\title{
Water Treatment with New Nanomaterials
}

\author{
Shahin Homaeigohar ${ }^{+}(1)$ \\ Nanochemistry and Nanoengineering, Department of Chemistry and Materials Science, \\ School of Chemical Engineering, Aalto University, Kemistintie 1, 00076 Aalto, Finland; \\ Shahin.homaeigohar@fau.de \\ + Current address: Institute of Biomaterials, Department of Materials Science and Engineering, \\ University of Erlangen-Nuremberg, 91058 Erlangen, Germany.
}

Received: 10 May 2020; Accepted: 25 May 2020; Published: 25 May 2020

check for updates

\begin{abstract}
The studies introduced in this special issue aim to provide a state-of-the-art vision for nanomaterials-based technology that could profit the water treatment industry. Given the expanding crisis of water shortages across the world, this perspective is invaluable and of paramount importance. No doubt, as the environmental challenges are going to be more complicated and to extend to as-yet unconsidered areas, we need to upgrade our facilities and knowledge to address them properly. Nanomaterials are indeed promising building blocks for such advanced technologies that enable them to purify water streams from complex pollutants in an energy, cost and time-effective manner. The focus of the (review and original research) articles collected in this issue is on various kinds of nanomaterials made of carbon, polymer, metal, and metal oxides (magnetic and photocatalyst), that are employed for adsorption and photodegradation of heavy metals and organic pollutants, respectively. Here, I briefly review the insights given in these precious studies and suggest new directions for future research in this field.
\end{abstract}

Keywords: nanomaterial; water treatment; adsorption; membrane; photocatalysis; nanohybrids; ecotoxicology

\section{Introduction}

While water shortage across the world are threatening the well-being of the human community, emerging advanced technologies targeted to address this challenge are promising. In this regard, nanomaterials have played a crucial role and offered new opportunities for the construction of permeable and selective membranes and adsorbents. Such features are of paramount importance, particularly given the limited available energy resources. In this issue, it was aimed to cover new researches dealing with water treatment based on nanomaterials made of polymer, metal, composite, ceramic (metal oxide), carbon, etc., that are shaped in various dimensionalities such as particle (0D), fiber (1D), and film (2D-3D).

In 2018, the special issue of "Water treatment with new nanomaterials" was introduced to the research community actively working on this subject all around the world. Following the call, within the course of running this issue, several articles were submitted to the editorial office, thereof seven were accepted for publication. These included five original research papers and two review papers. In the next sections, the published studies are briefly summarized.

\section{Review Papers}

Both reviews published in this issue give an overview of the potentials of nanomaterials for different water treatment approaches in a critical manner. Homaeigohar et al. [1] classified the nanomaterials studied for water purification based on the technologies benefitting from them, as nanosized adsorbents, 
nanomembranes, nanophotocatalysts, etc. They reviewed the relevant state-of-the-art approaches while pointing out to the potential bottlenecks. As an important aspect of this study, they discussed the fate of nanomaterials released into soil and water and their potential adverse impacts on the respective biota. As they report, according to the "ISI Web of Knowledge" statistics on the subjects discussed in this review, i.e., "Nanomaterials for Water Treatment" and "Environmental Impacts of Nanomaterials," 222 and 63 respective articles have been published from 2010 to 2020 . These numbers imply that the former topic has been paid more attention during the past ten years, while the second topic has been taken less seriously in the research community. Given the potential impacts and risks of nanomaterials on the environment, there is a need to allocate more time and budget to research on this critical topic.

Yaqoob et al. [2] reviewed various classes of nanomaterials studied for the sake of water treatment depending on their function. A particular emphasis has been given on nanophotocatalysts and their potential applications and operation mechanism, nano/micromotors, nanomembranes, and nanosorbents. The merits of this review could be its futuristic outlook on these classes of nanotechnolgies and its critical view while highlighting the available potentials.

\section{Original Research Papers}

Homaeigohar and Elbahri [3] reported on the synthesis of carbon buckypaper adsorbents made from amphiphilic (oxygenated amorphous carbon $\left(\mathrm{a}-\mathrm{CO}_{\mathrm{x}}\right) /$ graphite $(\mathrm{G})$ ) nanofilaments. Such a buckypaper is able to dynamically adsorb and separate organic biomolecules (i.e., urease enzyme) from water. Bearing in mind the dynamic nature of the test, this adsorbent has shown to be optimally efficient in adsorption of the enzyme (88\%) and at the same time to be notably permeable to water $\left(4750 \mathrm{~L} \cdot \mathrm{h}^{-1} \mathrm{~m}^{-2} \mathrm{bar}^{-1}\right)$; thus, assuring selectivity and permeability required for a low energy consuming water treatment.

Heydari et al. [4] studied and compared the relative efficiency of four groups of supported titanium dioxide $\left(\mathrm{TiO}_{2}\right)$ that were utilized for the purpose of photodegradation of 2,4-dichlorophenoxyacetic acid (2,4-D) in Killex ${ }^{\circledR}$, a commercially available herbicide. These four groups included floating $\mathrm{TiO}_{2}$ spheres, an anodized $\mathrm{TiO}_{2}$ plate with nanotube arrays, anodized $\mathrm{TiO}_{2}$ mesh with a $3 \mathrm{D}$ nanotube structure, and electro-photocatalysis using the anodized $\mathrm{TiO}_{2}$ mesh. Their energy consumption upon exposure to an ultraviolet light-emitting diode (UV-LED) light source at $\lambda=365 \mathrm{~nm}$ in a semi-passive mode was employed as an indicator for photocatalysis efficiency. Based on the authors' results, 3D nanotubes of $\mathrm{TiO}_{2}$ surrounding the mesh enable a higher photodegradation efficiency compared to 1D arrays on the anodized plate.

Zhang and Wang [5] synthesized nanoscale zero-valent iron (nZVI) particles via pulse electrodeposition and loaded them on the surface of biomass activated carbon (BC). The BC-nZVI composite was employed as an adsorbent for the removal of methyl orange (MO) from water. The adsorption test implied that the adsorbent is able to remove $97.94 \% \mathrm{MO}$ from water in one hour. This removal percentage can be optimally enhanced with a higher temperature, a larger BC-nZVI dosage, and a lower initial concentration of $\mathrm{MO}$ under neutral $\mathrm{pH}$.

Campagnolo et al. [6] developed an $\mathrm{Au} / \mathrm{ZnO}$ hybrid photocatalyst mounted on a poly (methyl methacrylate) (PMMA) fibrous substrate in a sequential manner. The fabrication process comprised the thermal conversion of the $\mathrm{ZnO}$ precursor already incorporated into PMMA electrospun fibers and then dipping of $\mathrm{ZnO} / \mathrm{PMMA}$ fibers into Au precursor solution and heating. The as-formed hierarchical heterogeneous photocatalyst was utilized for photodegradation of organic water pollutants, specifically methylene blue (MB) and bisphenol A (BPA), under UV light.

Ying et al. [7] synthesized a nanocomposite adsorbent system comprising meso- and microporous tire-derived-carbon support loaded with iron oxide nanoparticles. This nanocomposite adsorbent selectively adsorbed Se(IV) ions from simulated wastewater. The adsorption kinetics of this nanocomposite adsorbent was assessed in a fixed-bed setting and after several column runs by inductively coupled plasma-optical emission spectroscopy (ICP-OES), quantifying the concentration changes over time. Fitting the obtained data to a pseudo-second-order rate law, equilibrium values were 
determined that alongside the effluent concentration data could help the calculation of reaction constants and column coefficients through the Adams-Bohart model. As the authors believe, their findings allow the utilization of this nanocomposite adsorbent for fixed-bed column systems and enable further research based on mixed pollutants in real wastewater.

\section{Outlook and Future Directions}

Nanomaterials are promising building blocks for the next generation of water purification technologies and water pollution control systems. To expedite the realization of the nano-based water treatment approaches, there are still several milestones that need to be reached:

(1) Next generation of nanoadsorbents: Nanomaterials can be employed in the development of nanoadsorbents with different surface functionalities that optimally capture polar and non-polar pollutants from water. By using nanophotocatalysts, adsorption is coupled with photodecomposition. Therefore, the adsorbed organic pollutant is decomposed to harmless byproducts and the previously occupied surface becomes free for a subsequent adsorption/photodecomposition cycle. In relevance to the photocatalysis based water treatment, it is crucial to hamper hole-electron recombination in the photocatalyst and also to replace UV light with solar visible light as the main stimulus for the process. This latter target assures energy efficiency and more extensive usability of photocatalysis for water treatment. A critical bottleneck for the nanoparticulate adsorbents is their aggregation tendency and difficult recovery. To address these problems, they could be deposited on nanofiber substrates. Accordingly, not only is the aggregation level notably alleviated and the recovery of the nanoparticles is facilitated, but also their high availability to the external water medium is preserved.

(2) Next generation of nanomembranes: Nanomaterials can be also used as the building blocks of nanostructured membranes. Particularly, electrospun nanofibers and graphene nanosheets have been widely investigated in the last decade for such a purpose. Electrospun nanofiber mats offer remarkable potential for size exclusion and also adsorption of water pollutants. Owing to their adjustable pore size, extraordinary porosity, and open porous structure, they enable energy-efficient, thus economical, water treatment process. For this reason, they have been appealing for the construction of state-of-the-art ultra- (UF) and nanofiltration (NF) membranes as a permeable, solid support for the selective layer. Despite such merits, nanofibrous membranes have not yet been industrially realized. This issue might stem from the lack of proper and reliable testing of such membranes. Nanofibrous membranes must be tested over a long time period, and under the chemical, thermal, and mechanical conditions commonly found in practice and with real wastewater models. Unrealistically, at the lab scale, the nanofibrous membranes are typically tested in the presence of only one type of pollutant and co-existence of other dye, ionic, or organic pollutants, as found in real wastewater, is ignored. Graphene membranes i.e., those comprising mono-/few-layer graphene nanosheets also belong to the next generation of water membranes. They theoretically can offer remarkable water permeability, while providing the ionic selectivity that is comparable to classic NF and ideally reverse osmosis (RO) membranes. However, their potentials for water treatment have been predominantly proven theoretically rather than experimentally, and practical employment of such membranes would take time.

(3) Energy efficiency and scalability: There are currently several barriers against the widespread commercial use of nanomaterials for water treatment. These include technical shortcomings relating to scale-up and integration of nanomaterials into water purification technology, safety, cost, and energy effectiveness. For example, $\mathrm{TiO}_{2}$ nanoparticles and carbon nanotubes (CNTs) are of the most largely investigated nanomaterials for dye adsorption. Yet, their toxicity and costly production method involving high temperature and pressure are indeed discouraging for industrialization. Aside from that, $\mathrm{TiO}_{2}$ nanoparticles should be UV irradiated to photodecompose the dye molecules that raises the costs of the process. Therefore, as a prospect, the application of 
nanomaterials for water treatment is justified in case they can be produced at large amounts with reasonable costs, proportional to different classes of wastewaters.

(4) Sustainable and ecofriendly nanomaterials: The nanomaterials being used in the development of micro-, ultra-, and nanofiltration membranes can potentially be freed into water upon exposure of the membrane to aggressive water streams with intricate stress patterns. Thus, as an important priority, nanoparticles must be firmly stabilized on/in the membrane structure through physicochemical treatments. Moreover, nontoxic materials should be considered that are environmentally less challenging. In this regard, the new generation of nature-derived nanomaterials such as cellulose nanomaterials could be promising. Additionally, it is of utmost importance to develop synthesis and processing methods that are green and minimally involve hazardous chemicals. Short term investigations have implied that some nanomaterials are safe to human beings, plants, and animals. However, there is no guarantee for their long-term safety. Therefore, development of state-of-the-art water treatment systems based on nanomaterials should be pursued with caution. From the technological point of view, it is also crucial to properly design these systems so that they minimally release nanomaterials into the environment.

Funding: This research received no external funding.

Acknowledgments: The reviewers who kindly reviewed the submissions to this special issue and provided precious scientific inputs to the submitted articles are gratefully acknowledged.

Conflicts of Interest: The authors declare no conflict of interest.

\section{References}

1. Ghadimi, M.; Zangenehtabar, S.; Homaeigohar, S. An Overview of the Water Remediation Potential of Nanomaterials and Their Ecotoxicological Impacts. Water 2020, 12, 1150. [CrossRef]

2. Yaqoob, A.A.; Parveen, T.; Umar, K.; Mohamad Ibrahim, M.N. Role of nanomaterials in the treatment of wastewater: A review. Water 2020, 12, 495. [CrossRef]

3. Homaeigohar, S.; Elbahri, M. An Amphiphilic, Graphitic Buckypaper Capturing Enzyme Biomolecules from Water. Water 2019, 11, 2. [CrossRef]

4. Heydari, G.; Hollman, J.; Achari, G.; Langford, C.H. Comparative study of four $\mathrm{TiO}_{2}$-based photocatalysts to degrade 2, 4-D in a semi-passive system. Water 2019, 11, 621. [CrossRef]

5. Zhang, B.; Wang, D. Preparation of Biomass Activated Carbon Supported Nanoscale Zero-Valent Iron (Nzvi) and Its Application in Decolorization of Methyl Orange from Aqueous Solution. Water 2019, 11, 1671. [CrossRef]

6. Campagnolo, L.; Lauciello, S.; Athanassiou, A.; Fragouli, D. Au/ZnO hybrid nanostructures on electrospun polymeric mats for improved photocatalytic degradation of organic pollutants. Water 2019, 11, 1787. [CrossRef]

7. Ying, A.; Evans, S.F.; Tsouris, C.; Paranthaman, M.P. Magnetic Sorbent for the Removal of Selenium (IV) from Simulated Industrial Wastewaters: Determination of Column Kinetic Parameters. Water 2020, 12, 1234. [CrossRef]

(C) 2020 by the author. Licensee MDPI, Basel, Switzerland. This article is an open access article distributed under the terms and conditions of the Creative Commons Attribution (CC BY) license (http://creativecommons.org/licenses/by/4.0/). 